\title{
RELACIONES VIOLENTAS EN VOCI DE DACIA MARAINI
}

\author{
VIOLENT RELATIONSHIPS VOCI OF DACIA MARAINI
}

Begoña González

\section{Resumen:}

El objetivo de este artículo es un análisis de las difíciles relaciones que se establecen entre los personajes femeninos de una misma familia, madre e hijas, a causa de la violencia machista en la novela Voci de Dacia Maraini. Esta aparente novela negra, en realidad narra la violencia visible e invisible contra las mujeres, atrapadas en una red de secretos, mentiras y silencios para esconder una relación incestuosa por parte del padre.

\section{Palabras clave:}

personajes femeninos, violencia, Dacia Maraini, mujeres, abusos

\section{Abstract:}

The aim of this article is an analysis of the difficult relationships established between female characters within the same family, mother and daughters, due to the patriarchal violence in Dacia Maraini's novel Voci. This crime novel tells the story of visible and invisible violence against women, trapped in a web of secrets, lies and silences to hide an incestuous relationship on the part of the father.

\section{KeYWORDS:}

female, violence, Dacia Maraini, women, abuse 


\section{Voci de Dacia Maraini}

La novela Voci de Dacia Maraini se publica en 1994 y forma parte de la larga lista de obras en las que la escritora italiana trata el tema de la violencia contra las mujeres. A pesar de ello, la mayor parte de los análisis de la crítica literaria se centran en otros aspectos.

Según Patrizia Giuda (2017) se trata de un "giallo anomalo", es decir, una novela policiaca irregular, ya que no sigue las reglas específicas del género. Esto se puede apreciar en la presencia de un análisis psicológico de los personajes, algo que no sucede en la novela negra (Cannon, 2011). Se podría decir que el objetivo principal de la obra no es llegar a un público apasionado de la literatura policiaca, sino denunciar, a través de este formato, la impotencia social frente al feminicidio.

Barbara Kornacka (2018) considera Voci una novela sobre los sentidos. Es innegable que la voz es uno de los ejes principales para el desarrollo de la historia, dado que se trata del instrumento a través del cual una de las protagonistas trata de llegar a la verdad. El análisis de Kornacka se centra en la utilidad de las voces, la importancia de saber escuchar, y en cómo se llega al sentido del oído a través de la sonoridad de las diferentes voces que pertenecen a los personajes de la novela.

Ada Testaferri (2000), por otra parte, analiza la novela desde el punto de vista del estilo narrativo y la intertextualidad. Se centra sobre todo en las estructuras narrativas, aunque también tiene en cuenta la finalidad social de la obra. Considera que la escritora mezcla las características del género policiaco con el diario. Sostiene que se trata de una novela negra que sigue una perspectiva de género, con la cual la autora denuncia la impronta de la sociedad patriarcal.

Voci es una novela protagonizada por mujeres. Algunas de ellas, como la periodista Michela Canova o la comisaria Adele Sòfia, son mujeres independientes, pero cuyas profesiones las absorbe, desplazando su identidad a un campo masculino. Otras se presentan como dependientes y atadas a diferentes personajes masculinos, como la víctima Ángela Bari, una mujer misteriosa; su madre, que no sigue los estándares de la madre tradicional o su hermana, incapaz de despegarse de una infancia atormentada. Todas ellas, de una forma u otra, están relacionadas al estar sujetas a las dinámicas de dominación que el patriarcado reserva a las mujeres, bien a través de sutiles ataduras o a través de la violencia más feroz.

Tomando como punto de partida el asesinato de Ángela de Bari, desfilan diferentes personajes femeninos con los que se había relacionado. La víctima no solo ha sido privada de la vida sino también de su voz y son otras voces las que reconstruirán los detalles, a veces contradictorios, de su trayectoria, convirtiendo el libro en una especie 
de autobiografía relatada por otras personas. Su identidad se presenta cancelada, reconstruida solo en parte, pues sus parientes hablarán de su infancia, excluyendo el periodo de su edad adulta. De esta manera se subraya la trascendencia de esa etapa con respecto al asesinato de la protagonista en el presente. La investigación que llevará a desvelar su asesino, en realidad descubrirá que los abusos sexuales sufridos en la infancia la había anulado como sujeto; una violencia que se convierte en el lazo de unión con todas las demás mujeres participes de este relato.

\section{Personajes femeninos relacionados}

\subsection{Michela Canova}

Periodista en Roma, tiene que preparar, por encargo de su jefe en la radio, un programa sobre crímenes contra las mujeres. Sin embargo, a pesar de la existencia de muchos asesinatos, ella se concentra en un delito con el que se identifica inmediatamente, el homicidio de su vecina Angela Bari. La periodista inicia una investigación imparable, entrevistas con familiares, sospechosos, etc. Su trabajo se desarrolla paralelamente a las investigaciones de la policía, pero la implicación de la protagonista es tal que resulta difícil saber quién trabaja más de los dos.

El público lector conoce de la periodista dos aspectos: por un lado, un trabajo que la absorbe y, por otro, sus problemas personales. Se trata de una mujer involucrada en su profesión, siempre con prisas y acompañada de su grabadora para poder analizar las versiones que los sospechosos le proporcionan después de las entrevistas. De un día para otro, su vida cambia y gira en torno al asesinato de Angela Bari, de sus parientes o amigos. No tiene lazos familiares fuertes ni tampoco relaciones estables con hombres, quizás porque su imagen de la masculinidad no es muy positiva, a causa de la actitud machista de su padre y, como consecuencia, su sentimiento de animadversión hacia él: "Era un uomo così giovane anche da vecchio che, quando l'ho visto morto, non riuscivo assolutamente a crederci. [...] Eppure tante volte avevo desiderato ucciderlo. Per i suoi mille tradimenti, per la sua assoluta mancanza di riguardi nei confronti di mia madre, per il suo elegante e plateale egoismo" (Maraini, 2017: 28-29).

Tampoco tiene una buena relacion con su madre, cuyo modelo rechaza y cuya voz detesta: “è la voce di mia madre che mi parla all'orecchio; chissà perché l'ho tanto odiata quella voce, al punto da modificare la mia e renderla irriconoscibile, lontana da ogni area familiare" (Maraini, 2017: 157). Michela sufre de "matrofobia" que, como explica Adrienne Rich (1976), consiste en un profundo rechazo hacia la figura de la madre por miedo a convertirse o parecerse a ella. Especialmente, cuando la referencia que se tiene de la madre, como en este caso, es que trasmite a la hija los roles tradicionales y 
conservadores, además de su condición pasiva en la sociedad, siempre en un segundo plano detrás de la figura masculina.

La periodista muestra otro aspecto de su vida íntima: a veces menciona a Marco, con quien mantiene una relación a distancia por motivos de trabajo, y que contribuye a empeorar su consideración sobre las relaciones sentimentales con hombres: el hombre la ignora, evita comunicarse con ella y le genera una preocupación desmedida. Además, Marco es considerado sospechoso del asesinado de Ángela puesto que se conocían ya que al ser vecina de Michela habían coincidido en alguna ocasión, incluso se especula que podrían haber mantenido una relación sentimental.

Si bien la violencia se concentra en el asesinato de Ángela Bari, el personaje de Michela Canova también está sometido a una fuerte violencia simbólica, primero por sus relaciones familiares, luego por su relación sentimental $\mathrm{y}$, por último, por sus relaciones en su carrera profesional. Su jefe, cuando le encarga la realización de un programa basado en crímenes contra las mujeres, la trata ante todo con indiferencia y piensa en ella para ese trabajo porque es una mujer. La ocurrencia de este programa surge ante el incremento de mujeres que escucha la radio $y$, por tanto, ve la necesidad de capitalizar esta audiencia tratando temas que en general se denominan "temas" de mujeres o que gustan a las mujeres. Por lo tanto, sus ideas denotan un fuerte prejuicio de género: "niente politica, niente sport [...] le donne vogliono le storie, ha capito, Canova, le storie d'amore, naturalmente prima di tutto e poi di morte, di sofferenza, di terrore, ma hanno una fame cronica di storie" (Maraini, 2017: 17),

El jefe no se siente involucrado con el problema de la violencia, y su objetivo no es otro que el de aumentar la audiencia. Su retrato es el de un hombre sin escrúpulo que manipula ese tema en su propio beneficio económico: "non facciamo casi personali, ci vuole coralità, numero. Voglio statistiche precise, dati incalzanti" (Maraini, 2017: 84). En general esto puede asociarse al denominado "morbo periodístico". Según explica Parga (1997) la llamada "crónica roja", la que tiene que ver con las muertes o asesinatos, ha pasado de ser un medio que contribuye a la concienciación social sobre el problema a ser un asunto lucrativo. La excesiva visualización de la violencia según Parga, hace que esta se vea como algo común y, por tanto, se produzca un efecto rebote en el público.

Los medios se hacen eco de la muerte de Ángela y muestran sobre todo datos en relación con la brutalidad del asesinato. En un primer momento la prensa expresa sorpresa y rabia frente a un crimen de esas características. Sin embargo, según pasa el tiempo, empiezan a poner en entredicho su vida personal: "Ad una settimana dal suo assassinio i giornalisti ormai sono dentro con tutti e due i piedi nella vita di Angela e la calpestano senza riguardi: "come viveva se non aveva un lavoro fisso? Perché aveva 
orari così strani? È vero che ha fatto una parte in un film pornografico? [...] Si insinua facesse la prostituta" (Maraini, 2017: 25).

Dacia Maraini muestra a través de Ángela un comportamiento que se ejerce de manera habitual contra las víctimas de violencia machista. Al principio la sociedad se muestra conmovida, pero después hurga en sus vidas personales para encontrar posibles razones que justifiquen su muerte o que las culpabilicen. Los expertos describen este fenómeno como victim blaming; se trata de una acción desvalorativa, cuando la víctima de un crimen es la única responsable y por consiguiente la culpable del crimen que se ha cometido hacia ella. El ejemplo más difundido de este fenómeno aparece en los casos de violaciones en los que en general la víctima es señalada como culpable, porque viste de manera provocativa, seduce o pide de manera indirecta tener sexo (Coates et alii, 2006).

Maraini representa en esta relación entre Michela y su jefe el desequilibrio de roles y oportunidades entre mujeres y hombres que se ha transmitido a través de la cultural en la sociedad. Según Unger y Crawford (1996), se considera que las cualidades ideales esperadas en un hombre son la protección, la fuerza y la dominación, mientras que las que se esperan de una mujer son la debilidad, la sumisión y la dependencia. Esto puede asociarse a la intención del jefe de Michela, cuando considera que hay unos temas específicos de mujeres que se concentran en aspectos frívolos, macabros o intrascendentes, que nada tiene que ver con la cultura o la política, es decir, temas asociados a los intereses de los hombres y que sirven para ensalzar su presupuesta inteligencia.

Michela sufre un episodio de "micromachismo". Un día mientras trata de mover su Cinquecento, aparcado entre dos coches, un hombre la observa y la increpa, en cuanto representante de la incapacidad de la mujeres al volante: "Riprendo a manovrare, sudando, cercando di non toccare il suo prezioso paraurti. "Imbranata come tutte le donne!" sento che dice a mezza voce. Se ne sta in piedi, a braccia conserte, fissandomi con aria punitiva" (Maraini, 2017: 32). El episodio no es baladí y remite a la autoridad que los hombres ejercen sobre las mujeres de forma arbitraria y sin que entre ellos exista ningún tipo de relación parental o de otro tipo. El prejuicio sexista autoriza a los hombres a convertirse en "padres" simbólicos que sancionan o premian las acciones de las mujeres. La aceptación por parte de ellas viene determinada al tratarse conductas que se encuentran normalizadas dentro del imaginario social y cultural colectivo (Bonino, 1996). En el caso que nos ocupa, la persona que profiere el insulto, considera despectivamente que ella no es buena conductora, ya que la cultura y la tradición de muchas sociedades piensan que la conducción es un asunto de hombres.

Igualmente, podríamos relacionar esta situación con el fenómeno del mansplaining. Está claro que esta noción en general se asocia al hecho de interrumpir la voz de las 
mujeres en los espacios públicos, pero teniendo en cuenta que consiste en explicar a las mujeres como tienen que hacer las cosas, es frecuente, como le sucede a Michela, que los hombres quieran enseñar a las mujeres como conducir, como aparcar, etc. puesto que las consideran incapaces. Explica Kidd (2017) que este comportamiento está presente en la transmisión cultural y toca diferentes campos y situaciones.

2.2 Adele Sòfia

Adele Sòfia es la comisaria que se encarga de resolver el crimen de Angela Bari. Este personaje ya ha sido utilizado por Dacia Maraini en su novela Buio (1999), otra de sus obras cuya temática principal gira en torno a diversos abusos violentos contra mujeres, niños y niñas.

El público lector conoce a Sòfia a través de las impresiones de Michela, que la define del siguiente modo: "estroversa, diretta, e di mente fredda" (Maraini, 2017: 145). Las dos mujeres trabajan juntas indirectamente ya que la periodista necesita de la ayuda de la comisaria para preparar su programa. Igualmente, las entrevistas con los sospechosos secundan la investigación policial. Una relación que ante todo se consideraría profesional se convierte, con el desarrollo de la historia, en una amistad. Periodista y comisaria comparten diferentes momentos de sus vidas privadas. Sus conversaciones tienen lugar mientas que Sòfía prepara la cena o durante la misma. La siguiente cita muestra una de las ocasión en las que Michela reflexiona sobre la dualidad de la comisaria que debe actuar en lo público como hombre pero que recupera su feminidad en el ámbito privado: "Con quelle dita che conoscono sticita dei cibi, stringe le manette ai polsi degli assassini... Che sia prio della scienza materna questo mescolare... il nutriente al castigante" (Maraini, 2017: 153). La relación entre ambas mujeres es compleja: la inspectora se convertirá en cierto modo en la "madre simbólica" de Michela, una mujer mayor que ayuda y protege a otra más joven.

Dacia Maraini no muestra las emociones de la comisaria, es una mujer que se limita a hacer su trabajo sin jamás exponer sus sentimientos o sensaciones frente a los delitos, siempre manteniene una posición impasible. La ausencia de implicación emocional es algo frecuente en muchos ámbitos profesionales, ya sea de manera voluntaria o impuesta. La posición de la comisaria, nos hace pensar en una falta de solidaridad o empatía por su parte hacia las mujeres víctimas. Ella desarrolla su trabajo, de manera sistemática, siguiendo los procesos burocráticos y las leyes, que están por encima de cualquier valoración emocional. Tal indiferencia puede considerarse una falta de sororidad entre mujeres. En términos de Lagarde (2009), la comisaria no entra a formar parte de ese pacto de reciprocidad entre mujeres, que tiene presente que lo que le sucede a una puede pasarle a todas. Como sucede en muchos trabajos, el de la comisaria parece estar por encima de todo y de las personas en especial. A diferencia 
de Canova, no traspasa los límites de su trabajo, es decir, se queda en un ámbito masculino de percepción intelectual y teórica de la realidad, sin descender a un nivel afectivo o ponerse en el papel de las víctimas. Michela admira estas cualidades en ella, consideradas superiores en una mujer: "La guardo mettersi in piedi con maesta. Tutto in lei comunica tranquillita, pazienza, robustezza mentale ... con quella fredda passione per i teoremi da dimostrare che la anima" (Maraini, 2017: 135).

\section{3 ÁNGELA BARI}

La historia de Voci gira entorno a la muerte de Ángela Bari y como consecuencia, es el personaje sobre el cual el público lector tiene más datos. Lo que sabemos de ella es a través de la red de relaciones de los sospechosos y de los parientes que entrevista Michela Canova. Angela aparece en escena ya cadáver, en el momento en el que la portera de su edificio describe su asesinato: "Venti coltellate, una furia... e non l'hanno ancora preso, poveri noi" (Maraini, 2017: 8). Maraini, con esa descripción de ensañamiento, sugiere que tras esa muerte se esconde un crimen pasional lleno de incógnitas sobre la víctima.

La vida de Ángela va construyéndose a través de las diferentes versiones que proporcionan otros personajes de su entorno, gracias a las cuales logramos componer un puzle que nos permite descubrir la verdad. Además, también otros agentes sociales, como los medios de comunicación, hacen hipótesis sobre su vida que nos permiten analizar cómo se presenta la mujer asesinada desde una perspectiva externa. La madre, la hermana o el padrastro nos desvelarán en diversos momentos de la historia datos contradictorios y secretos que permiten descubrir aspectos sobre la víctima, que ayudan a sacar a la luz crudas realidades, pero también ponen de manifiesto la manipulación de las vidas de las mujeres víctimas de violencia de género. Estas quedan despersonalizadas y despedazadas por las interpretaciones, hipótesis, mentiras o tergiversaciones de quienes las reconstruyen, puesto que ellas ya no puede hablar por sí mismas, ni ofrecer ninguna interpretación de los hechos que las atañen directamente. Ángela Bari ha dejado de ser una persona para convertirse en un objeto de la curiosidad de unos, del trabajo de otros, del morbo de la mayor parte de la audiencia.

\subsection{Ludovica BARI, HERMANa de Ángela}

La hermana de Ángela, Ludovica Bari, a priori, parece una mujer con ganas de colaborar en la investigación, pero sin mostrar a penas ningún sentimiento de dolor por la pérdida de su hermana. Ludovica ofrece dos versiones en la historia, pues padece alteraciones psíquicas y, por lo tanto, desarrolla una percepción compleja de la realidad. En las entrevistas con la periodista describe la relación que tenía con su hermana de pequeña, a quien describe como una persona diferente y con dificultades para encontrar 
un lugar en la sociedad: "era fragile, disordinata, incapace di organizzarsi, È sempre stata così anche da piccola, povera Angela, arrivava tardi a scuola, studiava ma non imparava, si faceva cacciare dalla classe per colpe non sue, veniva bocciata un anno sè e uno no, insomma un disastro" (Maraini, 2017: 36).

Insiste en su versión de que Angela llamaba la atención de todos por sus rarezas, además añade que padecía dolencias psíquicas, habiendo pasado por una clínica especializada en tratamiento de enfermedades mentales. También habría tenido un aborto que habría contribuido a empeorar su estabilidad emocional.

Al final de la obra, Ludovica cambia su versión y afirma haber mentido en sus primeros encuentros con la periodista y entonces quiere contar la verdad. Admite ante todo haber sentido celos de su hermana cuando nació, y que este sentimiento se convirtió en envidia más adelante. .Ludovica aprecia que todos prefieren a Ángela por su belleza, mientras que ella queda relegada a un segundo plano: "Tutto è cominciato quando mia madre ha dato alla luce Angela e io avevo quattro anni; il mondo mi è caduto addosso [...] io ho cominciato a crescere storta, deforme, coltivando l'invidia e la gelosia... ma Angela, questa sorella bellissima, che faceva innamorare tutti, reagiva fuori da tutte le regole" (Maraini, 2017: 246).

Desvela posteriormente y con un tono más convincente, una atrocidad que tuvo lugar en su infancia. El compañero de su madre, su padrastro, abusaba de las dos hermanas. Se mostraba cariñoso pero les hacía vivir un infierno donde reinaban las amenazas: "Se parli, farò morire tua madre e tua sorella, stai attenta" (Maraini, 2017: 248). Explica también, que entre él y Angela había una especie de unión perversa, una relación de amor enfermiza que fue desarrollándose con el tiempo. Además añade que la madre, de hecho, era consciente de su existencia y aceptaba los abusos: "mia madre $\mathrm{mi}$ ha fatto capire che quello era il sacrificio necessario per tenerlo in famiglia, per mantenere la sua posizione, la sua benevolenza" (Maraini, 2017: 249).

Irene Intebi (2001) sostiene que el abuso sexual infantil es uno de los traumas psíquicos más intensos a los que un niño se pueda enfrentar, afectando a su desarrollo posterior, y añade: "Es posible comparar sus efectos al de un balazo en el aparato psíquico: produce heridas de tal magnitud en el tejido emocional, que hacen muy difícil predecir cómo cicatrizará" (Intebi, 2001: 173). En general, como explica GarcíaJaime (2013) el abuso sexual impide un desarrollo normal en la personalidad de los niños, dejando secuelas difíciles de afrontar. Además de las consecuencias físicas, hay diferentes problemas psíquicos y crónicos que se ven reflejados en una incapacidad para adaptarse a la sociedad (García y Raya, 1998).

Con la confesión de Ludovica se evidencia una violencia que estaba en un segundo plano. El lector se encuentra frente a un abuso sexual contra niñas dentro de la propia 
familia, temática que aparece en otras obras de Dacia Maraini como Amore rubato (2012) con su relato "la sposa segreta", La lunga vita di Marianna Ucrì (1990) o Bagheria (2004).

La posición de la madre, aunque nos resulte atípica, también suele ser la habitual en estos casos. En el análisis de Carvalho et alii (2009) se sostiene que las madres pueden experimentar diversas posiciones cuando tienen que hacer frente a los abusos de los hijos: algunas sienten una gran culpa por no haber podido proteger a sus hijos; otras no consiguen superar la existencia del abuso; otras sienten rabia o impotencia por no haber detectado a tiempo la violencia que han sufrido sus hijos. Sin embargo, explica Toledo (2010), existe otra reacción en las madres, que a veces son cómplices o responsables de los abusos, por diferentes motivos, personales, psicológicos o sociales. En estos casos, la madre acepta la situación ya que de lo contrario perdería unos supuestos beneficios que le proporciona su marido: una posición social y una compañía masculina. Por esa razón motiva a su hija a continuar y simula ignorar los hechos, como precio que pagar por mantener su posición en la sociedad.

Sin duda su relación con la figura física y simbólica del padre marca negativamente la vida de Ludovica, como también marca la de la periodista Michela, pues la violencia que sufrió en su infancia hizo de ella una víctima que perdura hasta el momento presente en el que convive con Mario Torres, otro hombre violento que la maltrata físicamente: "Quando si arrabbia mi riempie di lividi. Quelli che ha visto l'altro giorno sulle mie braccia...era stato lui" (Maraini, 2017: 197). Su retrato robot es el del típico agresor, caracterizado por una doble imagen: por un lado, amable, cariñoso y, por el otro, brutal: "Poi facciamo l'amore e mi ripete in continuazione che mi ama" (Maraini, 2017: 199).

\subsection{Augusta Bari, madre de Ángela}

Michela Canova se entrevista con Augusta Bari, quien habla de las relaciones de sus dos hijas en familia y en sociedad. La mujer se muestra distante con la periodista y su discurso es extraño e incompleto: "Ha la tendenza a lasciare le frasi a metà nonostante lo sguardo acuto e determinato. Gli occhi vagano sulle cose senza vederle e poi ammutolisce" (Maraini, 2017:103).

La madre describe a Ángela sin entrar en muchos detalles. Se centra especialmente en la infancia de la hija, etapa en la que ya tenía dificultades para relacionarse con los que la rodeaban debido a su carácter: “Da bambina era timida, quasi paralizzata dalla timidezza, non riusciva neanche a... crescendo è diventata più spigliata, ma sempre ha avuto delle timidezze che la torturavano... Non era una ragazza felice, anche se allegra, era, era" Maraini, 2017: 104). También describe la relación que tenía Angela con su padrastro, un vínculo afectuoso relatado de manera idílica en el que disfrutaban pasando tiempo juntos: “Angela e Glauco si amavano moltissimo, stavano sempre 
insieme, andavano a fare gite in motocicletta, su per le montagne, a nuotare, a sciare, erano due sportivi meravigliosi" (Maraini, 2017: 108).

La madre muestra una gran impasibilidad ante el asesinato de su hija, resultando impactante su indiferencia, sin ni siquiera estar pendiente de la investigación sobre el asesino de Ángela: "I morti, secondo me, bisogna lasciarli in pace. Che importanza ha sapere chi è stato quando ormai lei non può tornare in vita? [...] Non voglio sapere chi ha ucciso mia figlia, non servirebbe a niente" (Maraini, 2017: 103-104).

Augusta no relata los episodios de violencia que su compañero había ejercido en la infancia de sus hijas, lo que confirma que ella apoyaba esos actos. Deja claro que se trata de una mujer que no sigue el modelo normalizado de madre protectora y afectuosa.

2.6. Glauco Elia, padre de Ángela

Glauco Elia, padrastro de Angela y Ludovica es sospechoso del crimen. En un primer momento también describe las difíciles relaciones de Angela cuando era pequeña y convivía con su madre. La describe como una niña difícil, incluso agresiva. Insiste en destacar que tenía ciertas manías y particularidades que no dejaban indiferente a nadie. Sin embargo, hace un comentario sexual en relación a la vida privada de Ángela que llama la atención de la periodista: "certe libertà eccessive, certi comportamenti disinvolti, secondo me più provocatori che altro" (Maraini, 2017: 268).

Al final de la obra, el señor Elia envía una grabación decisiva a la periodista Michela, donde desvela el pasado cruel y perverso que habría tenido lugar durante la infancia de sus hijastras y que encajaría con la última versión ofrecida por Ludovica.

En la primera parte de la narración el hombre se muestra como un salvador de la familia tras llegar a la vida de la madre de las niñas. Él ostenta la figura del hombre que le faltaba a aquella casa. Como se considera en la tradición patriarcal, es necesaria la presencia de esta figura masculina para marcar las directrices, una persona con poder de mando que establezca una serie de modelos de comportamiento. Elia se enorgullece de haber establecido en la familia relaciones que "domesticaron" a las dos niñas, algo por lo que su mujer le estaba agradecida. Mas adelante hace una distinción entre las hermanas, describiendo a Ludovica como mentirosa y altanera: “Da quando ha preso ad odiarmi perché le impedivo di comportarsi da prostituta ha inventato le cose più ignobili sul mio conto; e insisteva perché sua madre la credesse" (Maraini, 2017: 271). Ángela era para él todo lo contrario, contradiciendose así con su discurso inicial: "una creatura malleabile e tenera, sensuale e vogliosa di piacere [...] mi è stata fedele e complice fino all'ultimo" (Maraini, 2017: 271). 
Añade, una descripción enfermiza del cuerpo de Ángela, que expresa un deseo enfermo, perverso, obsesivo, una especie de fijación:

Chiunque di fronte al suo corpo, vestito o nudo che fosse, era preso da una voglia spasmodica di toccarlo, di carezzarlo, di penetrarlo, perfino di forzarlo, perché lei in qualche modo chiedeva proprio questo, voleva l'urto, la presa di possesso, l'invasione... per poi magari respingerti con ripulsa infantile... faceva no con la testa mentre le labbra, i seni dicevano di sì, offrendosi e negandosi nello stesso tempo con una sensualità che metteva addosso la voglia di uccidere (Maraini, 2017: 274).

Maraini se hace eco de la ideología patriarcal con el objetivo de denunciarla. "Presenta un fuerte contraste entre agresor y víctima con el intento de recoger la aporía del comportamiento de las mujeres. Su objetivo también es adentrarse en los meandros de la consciencia masculina para evidenciar los impulsos agresivos y animalescos" (Moreno, 2019: 93). Este es el caso de Glauco, que describe su crimen descargando la culpa sobre la víctima como resultado de una extraña posesión y obsesión que el cuerpo de la víctima ejercía sobre él. Sus palabras revelan cómo la violación y el asesinato están arraigados en la concepción simbólica de lo paterno, que controla y subyuga los cuerpos femeninos que considera sus pertenencias.

\section{Conclusiones}

A través del cadáver de Ángela Dacia Maraini representa lo que se considera el iceberg de la violencia: lo que el lector ve en un primer plano es su asesinato, pero bajo esa punta se esconde un pasado de abusos infantiles ejercidos por un miembro de su familia. Por otra parte, la historia muestra a Ludovica como el personaje a quien más han afectado los abusos sexuales, puesto que no se menciona que estos hayan causado un trauma en Ángela; al contrario, tanto ella como el padrastro hablan de aceptación de los abusos por parte de la víctima, incluso de la existencia de una relación consentida entre ellos. Queda claro que la relación perversa que establece con su padre hace de ella una mujer extraña, amada y odiada a la vez e, indirectamente, culpable de su propia muerte.

Cada personaje femenino está atrapado en una red de relaciones patriarcales que tiene validez para cualquier mujer del mundo. En primer lugar las relaciones de violencia simbólica, que afectan a Michela Canova y a Adele Sòfia, en ambientes donde aparentemente existe igualdad entre hombres y mujeres, que realizan los mismos trabajos. En segundo lugar, las relaciones de violencia física que padecen las hermanas Bari y que llevadas a sus extremas consecuencias procuran la muerte de Ángela. En tercer lugar, la falta de relaciones, empatía o la indiferencia social hacia este problema que afecta especialmente a las propias mujeres, como la comisaria, que no muestra ni emociones ni solidaridad. La violencia institucionalizada y normalizada, a través de 
los medios de comunicación despojan a las mujeres de su individualidad y humanidad para convertirlas en rumores, chismes, objetos de visión, y por tanto, productos de consumo.

Las diferentes voces de mujeres que se van subsiguiendo en el texto, devuelven al cadáver mutilado de Ángela su dignidad de persona. Ejemplifican las relaciones de sororidad entre mujeres, en las que unas reconstruyen la existencia de otras, rompiendo "el muro del silencio en el que las mujeres se han visto confinadas por una cultura que desprecia sus palabras, las banaliza o las ridiculiza" (Arriaga, 2020, p. 45). Muchas veces a lo largo de la novela se les aplica a los diferentes personajes femeninos la palabra "incompetente", no solo a Michela por parte de su jefe y por quien quiere ayudarla a sacar su coche del aparcamiento, sino también es el adjetivo que se aplica a la víctima. Ambas, por tanto, están relacionadas a través de la mirada patriarcal que utiliza el mismo rasero para todas las mujeres, sin tener en cuenta lo diferentes u opuestas que sean. A pesar de ello, Michela se identifica con Ángela y se convierte en su doble: "Mi sembra di conoscerla cosi bene, eppure non so niente di lei. E il fatto che sia stata cosi brutalmente straziata mi sembra improvvisamente una offesa fatta a me personalmente" (Maraini, 2017: 30). Es esta relación de sororidad la que conduce a visibilizar la violencia y a resolver el asesinato.

\section{REFERENCIAS BibLIOGRÁficAS}

Arriaga Flórez, M., "Literatura y mujeres. Querella, genealogías y Sororidades”, Aquelarre. Mujeres y medios de comunicación en la cultura de masas, entre la opresión y la lucha por la igualdad. Sevilla, Advook, 2020, pp. 39-62.

Bertone, M. y Meazzi, B., Curiosa di mestiere. Saggi su Dacia Maraini. Pisa, Edizioni ETS, 2017.

Cannon, J. "Voci and the Conventions of the Giallo." Italica n. 78, vol. 2, (2001), pp. 193-202. Carvalho, Q. C. M., Galvão, M. T. G., \& Cardoso, M. V. L. M. L. (2009). “Abuso sexual infantil: Percepción de las madres frente al abuso sexual de sus hijas". Revista Latino-Americana de Enfermagem, n. 17, vol 4, (2009), pp. 501-506.

Coates, L., Richardson, C., \& Wade, A., Reshaping Responses to Victims of Violent Crime. Presented at Cowichan Bay. Canada, B.C., 2006.

Diaconescu-Blumenfeld, R. \& Testaferri, A., The pleasure of writing: Critical Essays on Dacia Maraini. West Fafayette, Indiana, United states of America, Purdue UP, 2000.

García-Jaime, R., “Abuso sexual en la niñez”. Boletín Científico Sapiens Research, n. 3 vol 2, (2013), pp. 13-17.

García, R., Raya, A., Aplicación de los principios sistémicos como agentes preventivos de las relaciones incestuosas. Tesis de licenciatura. UNAM campus Iztacala, 1998. 
Giuda, P., "Il corpo e le voci”, Curiosa di mestiere. Saggi su Dacia Maraini. Bertone, M. y Meazzi, B. (eds). Pisa, Edizioni ETS, 2017.

Intebi, I. V., Abuso sexual infantil: en las mejores familias. Ediciones Granica SA, 2001.

Kidd, A. G., Mansplaining: The Systematic Sociocultural Silencer. Thesis doctoral, University of North Georgia, 2017.

Kornacka, B., "La scrittura udibile-alcune osservazioni sul romanzo Voci di Dacia Maraini". Écho des études romanes. Revue semestrielle de linguistique et littératures romanes, n. 2, (2006), pp. 71-87.

Lagarde, M., "La política feminista de la sororidad". Mujeres en Red, el periódico feminista, n. 11, (2009).

Maraini, D., Voci, Milano, BUR, 2017.

Moreno Lago, E. M. "Un teatro de denuncia: estrategias escénicas en la dramaturgia breve de Dacia Maraini”. Revista de la Sociedad de los italianistas españoles, n.13, (2019), pp. 8596.

Parga, J. S., "De la crónica roja al morbo mediático". Chasqui. Revista Latinoamericana de Comunicación, n. 60, (1997), pp. 4-8.

Toledo, R. T., "Las Madres Frente al Abuso Sexual Infantil Intrafamiliar de sus Hijos ¿Son Víctimas?", Revista Trabajo Social, n. 9, (2009), pp.1-19. 